\title{
To compare the blood pressure and heart rate during course of various types of anesthesia in wistar rat: A novel experiences
}

\author{
Jagdish Narayan', Pradeep Kumar², Ankit Gupta ${ }^{3}$ and Sunita Twari ${ }^{4}$ \\ ${ }^{1}$ Associate Professor, Department of Physiology, KG Medical University, Lucknow India, ${ }^{2}$ Professor, Physiology, KG \\ Medical University, Lucknow India, 3Junior Resident, Department of Physiology, KG Medical University, Lucknow India, \\ ${ }^{4}$ Professor and Head, Department of Physiology, KG Medical University, Lucknow India
}

Background: Rats are commonly used animals in development of newer drugs, rectification of toxicity and to record the various alterations in physiological parameters following pharmacological and non pharmacological interventions. Aim and Objectives: The purpose of this study was to identify the best physiological window during anesthesia. Therefore, we compared the effect of anesthesia using combination of ketamine and xylazine (KX) and thiopental sodium (intraperitoneally) on blood pressure and heart rate in adult male Wistar rats. Material and Methods: Twelve, male Wistar rats with a mean body weight of $260 \pm 15 \mathrm{~g}$ were acquired. Thiopental sodium and cocktail of ketamine and xylazine (KX) were administered (ip) in group- I and group-II respectively. The systolic blood pressure and heart rate was recorded in both the groups till the awakening phase. Results: We found that there was a constant SBP and HR in Ketamine/Xylezine groups that are from 30 to 90 minutes after injection of anesthesia while this window was not observed in thiopental group. Conclusion: Our study concludes that the best time to observe the effect of newer drug during period between 30-90 minutes after anesthesia.

Key words: Anesthesia; Ketamine; Xylezine; Blood pressure wister rats

\section{Access this article online}

Website:

http://nepjol.info/index.php/AJMS DOI: 10.3126/ajms.v9i6.20625 E-ISSN: 2091-0576

P-ISSN: 2467-9100

\section{INTRODUCTION}

The rodents including rat are commonly used animals in development of newer drugs, rectification of toxicity and to record the various alterations in physiological parameters following pharmacological and non pharmacological interventions. Safe and reliable anesthesia is required or recommended in almost all type of experimental procedures. ${ }^{1,2}$ Since a given anesthesia may affect experimental outcomes, the choice of a suitable anaesthesia has to be considered carefully in respect to the particular experiment. Usually, it is necessary to keep some factors in mind before giving the anesthesia. These factors include low stress during induction of anesthesia, the least impact on physiological parameters and recovery period of anesthesia. Advantages and disadvantages of various anesthetics administered by different route were studied and reported that the use of isoflurane (ISO) requires specialized equipment as it is an inhalational anesthesia. ${ }^{3-5}$ Thiopental sodium is a rapid-onset short-acting anesthetic and has been used commonly in the induction phase of general anesthesia in all laboratory animals. ${ }^{6}$ Advantages of thiopental sodium are pleasant and rapid induction as well as recovery from anesthesia. However, thiopental sodium reported to be a poor muscle relaxant, week analgesic activity and must be used with other agents. ${ }^{7}$ The effect of various anaesthetics has been demonstrated on respiratory parameters, body temperature, blood indices and cognitive functions using various animal models. ${ }^{8-10}$ With best of my knowledge there are very scared studies regarding effect of 
various anesthetic on variability of blood pressure during different phases of anesthesia in hypertensive rats. The purpose of this study was to identify the best physiological window during anesthesia. Therefore, we compared the effect of anesthesia using combination of ketamine and xylazine (KX) and thiopental sodium (intraperitoneally) in adult male Wistar rats on blood pressure and heart rate.

\section{MATERIAL AND METHODS}

Twelve, male Wistar rats with a mean body weight of 260 $\pm 15 \mathrm{~g}$ were acquired from IITR Lucknow India. The study was approved by institutional ethics committee. These animals were housed in groups of two in each cage at the animal facility of the King George's Medical University, Lucknow (UP). Cages specially made for laboratory animals containing a wooden bedding material for hygiene and safety. Cage bedding were changed twice weekly to avoid unwanted contamination. The rats were allowed to take commercially available diet (rat chow) and water ad libitum. The animal room was maintained at $25 \pm 2^{\circ} \mathrm{C}$ and there was an air change at least 15 cycles/hour. Twelve hour day night cycle was maintained by natural ways.

\section{Animals}

Animals received care according to the principles of India's Committee for the Purpose of Control and Supervision of Experiments on Animals (CPCSEA) guidelines. (Approval No.31/IAH/Pharma/14 dated 17/05/14 No. 55/ IAEC/2014)

Animals were divided in two groups: Group-I $(n=6)$ and Group-II $(n=6)$. Thiopental sodium and cocktail of ketamine and xylazine (KX) were administered (ip) in Group-II and Group-I respectively. The systolic blood pressure and heart rate was recorded in both the groups till the awakening phase.

\section{Recording of parameters}

Systolic blood pressure and heart rate was recorded by Non Invasive Blood Pressure machine with advanced software. (AD Instruments Australia) All the animals were acclimatized to the restrainer and tail cuff before recording of baseline parameters and experimentation. The animals were kept in the restrainer for at least 10 to 15 minutes daily for 5 days. Acclimatized animals provide faster and accurate BP measurements than non-acclimatized animals.

After allowing the animals to acclimatize to the restrainer, tail was introduced into the tail-cuff of the NIBP instrument and pulse transducer was placed distally to the tail-cuff over the tail. During measurement of blood pressure, it was ensured that rat never have its head bent sideways or its body compressed against the back hatch.

\section{Anesthesia}

Doses of Ketamine $(50 \mathrm{mg} / \mathrm{kg}$ ) and Xylazine $(5 \mathrm{mg} / \mathrm{kg})$ and Thiopental sodium $(50 \mathrm{mg} / \mathrm{kg}$ body wt) was calculated according to the weight of each rat and then ketamine/ xylazine cocktail mixture and thiopental sodium was prepared in a syringe and given through intra-peritoneal route to the respective groups under proper hygiene and precaution. Systolic blood pressure and heart rate was further recorded after anesthesia at an interval of 30 minutes.

\section{Statistical analysis}

The statistical analysis was done using SPSS (Statistical Package for Social Sciences) Version 15.0 statistical Analysis Software. The values were represented in Number $(\%)$ and Mean \pm SD. P value less than 0.05 considered as significant.

\section{RESULTS}

\section{Observation and results}

In the course of experiments the rats after anesthesia showed a good quality blood pressure signal as compared to conscious animals. The blood pressure signals were optimum at proximal part of tail. There were no significant differences in baseline values among the group-I and group-II. Our data showed that the systolic blood pressure increases from baseline $(117.82 \pm 4.42 \mathrm{mmHg}$ ) to $131.20 \pm 8.25 \mathrm{mmHg}(\mathrm{p}<0.05)$ in group-I. This increase was also observed in group-II. The systolic blood pressure found to be relatively constant between periods from 30 minutes to 90 minutes after anesthesia followed by decrease in blood pressure during awakening phase in group-I in which ketamine -Xylezine was given. (Table-1) It was observed that this pattern was different in group-II (thiopental group) and the decrease in systolic blood pressure was more evident at awakening phase. The base line heart rate was $290.44 \pm 12.24$ which increased $24 \%$ during initial phase of anesthesia followed by a constant phase and increased at the time of awakening in group-I. The decrease in $\mathrm{Hr}$ was throughout the anesthesia except in awakening phase in group-II. (Table-1)

\section{DISCUSSION}

Animal experimentation is the need of modern experimental science of medicine. Almost all the animal model required or recommended anesthesia to conduct proper experimentation as per guideline of CPCSEA. Various studies reported the effect of various anesthesias on different physiological parameters including systolic blood pressure and heart rate. Previous data revealed that there are significant effect of anesthetic on systolic blood pressure and heart rate. So, while demonstrating the effect of any newer drug (Phase-I- trail) it is necessary to eliminate or 
Table 1: Effect of two types of anesthesia on SBP and HR

\begin{tabular}{|c|c|c|c|c|c|}
\hline \multirow{2}{*}{$\begin{array}{l}\text { Parameters } \\
\text { Time }\end{array}$} & \multicolumn{2}{|c|}{ Heart rate (beat per minute) } & \multicolumn{2}{|c|}{ Systolic blood pressure (mmHg) } & \multirow[t]{2}{*}{$P$ value } \\
\hline & Group-I & Group-II & Group-I & Group-II & \\
\hline $\begin{array}{l}\text { Baseline } \\
\text { After anaesthesia }\end{array}$ & $290.44 \pm 12.36$ & $288.28 \pm 13.12$ & $117.82 \pm 4.47$ & $115.20 \pm 6.44$ & NS \\
\hline 5 minutes & $362.00 \pm 13.18$ & $364.00 \mathrm{v} 15.28$ & $131.20 \pm 8.25$ & $128.20 v 6.11$ & $P<0.05$ \\
\hline 20 minutes & $320.00 \pm 20.10$ & $325.12 \pm 19.20$ & $126.10 \pm 7.47$ & $120.00 \pm 3.22$ & $P<0.05$ \\
\hline 30 minutes & $250.67 \pm 13.3$ & $255.10 \pm 8.8$ & $122.0 \pm 6.00$ & $118.20 \pm 10.20$ & $P>0.05$ \\
\hline 60 minutes & $245.67 \pm 14.14$ & $240.00 \pm 13.20$ & $123.04 \pm 6.86$ & $116.25 \pm 7.2$ & $P<0.05$ \\
\hline 90 minutes & $248.20 \pm 4.20$ & $235.84 \pm 14.2$ & $122.00 \pm 3.2$ & $110.00 \pm 4.8$ & $P<0.05$ \\
\hline 120 minutes & $307.20 \pm 8.71$ & $239.90 \pm 12.22$ & $108.0 \pm 4.3$ & $98.20 \pm 4.8$ & $P<0.05$ \\
\hline
\end{tabular}

nullify the effect of anesthesia on systolic blood pressure and heart rate. Previous studies showed various changes by the anesthetics on physiological parameters in animals. In present study we identified the period during anesthesia in which least variation in SBP and heart was observed.

\section{CONCLUSION}

In our experiment, we compare the effect of anesthesia (thiopental sodium and Ketamine/Xylezine) and found that there was a constant SBP and HR in Ketamine/Xylezine groups that are from 30 to 90 minutes after injection of anesthesia while this window was not observed in thiopental group. Therefore the best time to observe the effect of newer drug during period between 30- 90 minutes after anesthesia.

\section{REFERENCES}

1. Krinke GJ. The Laboratory Rat. London: Academic Press; 2000.

2. Arras $M$, Autenried $P$, Rettich $A$, Spaeni $D$ and Rülicke $T$. Optimization of intraperitoneal injection anesthesia in mice: drugs, dosages, adverse effects, and anesthesia depth. Comparative medicine 2001; 51(5):443-456.
3. Tremoleda JL, Kerton A and Gsell W. Anaesthesia and physiological monitoring during in vivo imaging of laboratory rodents: considerations on experimental outcomes and animal welfare. EJNMMI Res 2012, 2:44.

4. Hildebrandt IJ, Su H and Weber W. Anesthesia and other considerations for in vivo imaging of small animals. ILAR J 2008, 49:17-26.

5. Prudian F, Gantenbein M, Pelissier AL, Attolini L and Bruguerolle B: Daily rhythms of heart rate, temperature and locomotor activity are modified by anaesthetics in rats: a telemetric study. Naunyn Schmiedebergs Arch Pharmacol 1997, 355:774-778.

6. Erhan E, Ugur G, Gunusen I, Alper I and Ozyar B. Propofol - not thiopental or etomidate - with remifentanil provides adequate intubating conditions in the absence of neuromuscular blockade. Canad J Anaesth 2003;50: 108-115.

7. Chuan-Xiu $Y$ and Tian-Le $X$. Thiopental inhibits glycine receptor function in acutely dissociated rat spinal dorsal horn neurons. Neurosci Lett 2005; 397: 196-200.

8. Meyer RE, Richard E and Fish RE. Anesthesia and analgesia in laboratory animals. Elsevierinc 2008. Second edition.

9. Avsaroglu $H$, van der Sar AS, van Lith HA, van Zutphen LF and Hellebrekers LJ. Differences in response to anaesthetics and analgesics between inbred rat strains. Lab Anim 2007, 41:337-344.

10. Stokes EL, Flecknell PA and Richardson CA. Reported analgesic andanaesthetic administration to rodents undergoing experimental surgical procedures. Lab Anim 2009; 43:149-154.

\footnotetext{
Authors Contribution:

JN- Reviewed the literature, manuscript preparation and critical revision of the manuscript; PK- Concept and design of the study, collected data and helped in preparing first draft of manuscript; AG- Collected data, literature search, statistically analyzed and interpreted; ST- Revision of first draft of manuscript and critical revision of the manuscript.

Work attributed to:

Department of Physiology, KG Medical University, Lucknow India.

ORCID ID:

Dr. Pradeep Kumar- ㄴ https://orcid.org/0000-0001-6918-4782

Source of Support: Nil, Conflict of Interest: None declared.
} 